\title{
Analysis of Energy Efficient Clustering Scheme in Wireless Sensor Network
}

\author{
Manpreet Kaur Aulakh \\ Assistant Professor, Dept of Computer Science, SBSSTC Ferozepur, India
}

\begin{abstract}
Wireless sensor networks are remote networks and works in adhoc manner. Sensor collects the information sensed by the self and sends it to cluster head created in clusters. Further cluster heads use to send this information to the sink where data fetched use to complied and processed according to application used. In our research, we will propose a novel method for establishing reliable and efficient data transmission in wireless sensor networks. In this approach, we will achieve energy efficiency by using hierarchical clustering method to form clusters in the sensor network. Network lifetime is increased by using this technique. To increase reliability and to utilize energy much more effectively multiple mobile sinks are used along with base station. Mobile sink nodes are used to enhance the performance metrics. Mobile sinks will be bring into grid area so that less energy consumption will be there for cluster heads. Mobile sinks will fetch data from cluster heads with minimize energy consumption. Grid will be divided into equal portions to form a uniform distribution of mobile sink. Particularly this paper analyze the various clustering techniques with mobile sink concept.
\end{abstract}

Keywords: Wireless Sensor Nodes, Mobile Sink, Leach Protocol, Multi-hop Communication.

\section{INTRODUCTION}

The recent technological advancements in the field of over an area of interest for collecting information. [3]The micro electrical mechanical systems (MEMS) have made decrease in size and cost of the sensor nodes has made it the manufacturing and use of small, low powered and possible to have a network of large number of sensor moderate cost micro-sensors[1] both technically and nodes, thereby increasing the reliability and accuracy of economically feasible. A Wireless Sensor Network (WSN) data as well as the area of coverage. Due to the low-cost [2] Consists of hundreds to thousands of low-power multi- deployment, the nodes are generally deployed with greater functioning sensor nodes, operating in an unattended degree of connectivity. Such redundancy also increases the environment, having capabilities of sensing, computation network fault tolerance as the failure of a single node has and communications. Wireless Sensor Networks are used negligible impact on the entire network operation. [4] for monitoring and collecting information from an LEACH assumes a simple model for the radio hardware unattended environment and for reporting events to the energy dissipation where the transmitter dissipates energy user. They monitor physical or environmental conditions to run the radio electronics and the power amplifier, and such as temperature, humidity, pressure, sound, vibration the receiver dissipates energy to run the radio electronics. etc.

For the experiments described here, both the free space and the multipath fading channel models were used, depending on the distance between the transmitter and receiver [6].

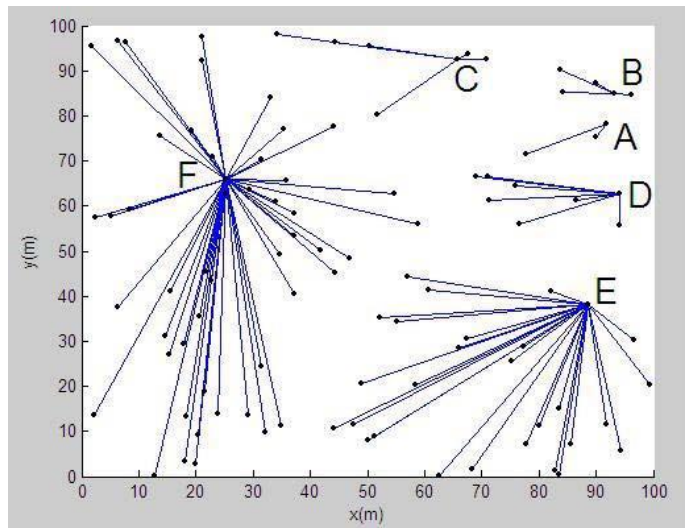

Since a sensor node is limited in terms of sensing and computation capacities, communication performance and power - a large number of sensor nodes can be distributed 
Vol. 5, Issue 3, March 2017

\section{PARAMETERS FOR COMMUNICATION IN WSN}

A. Node Deployment

Node deployment in WSNs is application-dependent and can be either manual or randomized. In manual deployment, the sensors are manually placed and data is routed through predetermined paths. However, in random node deployment, the sensor nodes are scattered randomly, creating an adhoc routing infrastructure. If the resultant distribution of nodes is not uniform, optimal clustering becomes necessary to allow connectivity and enable energy-efficient network operation. [7]

\section{B. Data reporting method}

Data reporting in WSNs is application-dependent and also depends on the time criticality of the data. Data reporting can be categorized as either time-driven, event driven, query-driven, or a hybrid of all these methods. The timedriven delivery method is suitable for applications that require periodic data monitoring. As such, sensor nodes will periodically switch on their sensors and transmitters, sense the environment, and transmit the data of interest at constant periodic time intervals. [3]

\section{Node/link heterogeneity}

In many studies, all sensor nodes were assumed to be homogeneous. However, depending on the application a sensor node can have a different role or capability. The existence of a heterogeneous set of sensors raises many technical issues related to data routing. For example, some applications might require a diverse mixture of sensors for monitoring temperature, pressure, and humidity of the surrounding environment, detecting motion via acoustic signatures, and capturing images or video tracking of moving objects. [6]

\section{Fault tolerance}

Some sensor nodes may fail or be blocked due to lack of power, physical damage, or environmental interference. The failure of sensor nodes should not affect the overall task of the sensor network. If many nodes fail, medium access control (MAC) and routing protocols must accommodate formation of new links and routes to the data collection BSs. [7]

\section{III.MOBILE SINK COMMUNICATION}

The consumption of energy in reception and sensing is independent from transmission distance. Energy consumption in computation is negligibly small compared to energy dissipation in other process such as sensing, transmission and reception of a bit as shown by Hoang and Motani [9]. Intra-cluster traffic can be varied if we change the size of the cluster accordingly by varying the number of nodes in a cluster. A change in a cluster size and the change in number of nodes will result in change in traffic load on a cluster head of that cluster. [10] If we increase the cluster size we can also accommodate more number of nodes. Therefore load on cluster head increases which may lead to depletion of the residual energy of the cluster head more rapidly and vice versa. Inter-cluster traffic means the data transfer between adjacent cluster heads on its way to the base station. The farthest cluster head from the base station have only its own data but the cluster head following it towards base station has to forward its own traffic and the traffic it received from its neighbor nodes. Thus, the cluster head which is nearest to BS will be dealing with maximum data traffic. Also larger the size of the cluster the cluster head requires higher energy to directly transmit or relay data to its adjacent cluster head on its path to the base station and smaller the size of the cluster, lesser will the amount of energy spent in transmitting it to the next hop cluster head or to the base station. We assume that energy spent at each node is consumed on reception and transmission only as energy spent on processing is negligible. [11]

\section{IV.CLUSTERING PROTOCOL WITH VIRTUAL FORCE}

This paper explained general framework to address two major issues in clustering protocols for WSNs: the network lifetime and the sensing coverage. This framework uses the principles of Virtual Field Force (VFF) [5] to determine the locations of the nodes within each cluster, in order to maximize the sensing coverage and minimize the energy consumption of these nodes, which in turn maximizes the lifetime of the network and extends its usability. The proposed framework is applied to the LEACH clustering protocol [10]. The new clustering protocol is called LEACH-VF (LEACH with Virtual Force).

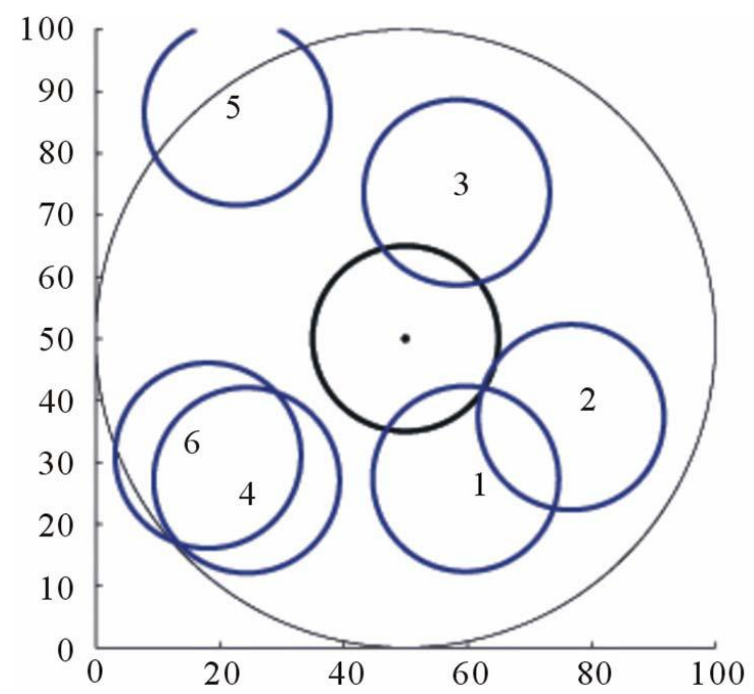

Fig. 3. Problems with the LEACH-based clustering

To explain the idea of the LEACH-VF protocol and present its potential advantages, consider the simple LEACHbased cluster with six sensor nodes shown in Fig 3. The outer large circle represents the cluster area and the small 
Vol. 5, Issue 3, March 2017

circles indicate the sensing coverage of each sensor node. The circle in the middle of the cluster represents the sensing coverage of the $\mathrm{CH}$. There are four problems with this cluster caused by the random distribution of nodes and the random selection of CHs:

1) There are areas with overlapped sensing coverage (i.e., areas covered by more than one sensor node). For example, sensor nodes 4 and 6 have a relatively large sensing coverage overlap.

2) There are areas with sensing holes (i.e., areas with no sensing coverage).

3) Some sensor nodes have coverage outside the cluster area. For example, sensor node 5 .

4) Some sensor nodes are located relatively far from their $\mathrm{CHs}$, while they can be relocated in closer places and still be useful to the cluster at a lower energy cost.

When the proposed LEACH-VF algorithm is applied to this cluster, the result is shown in Fig 4.

It is worth noting that the above-mentioned problems were resolved. Thus, the LEACH-VF algorithm takes a regular LEACH-based cluster as input and produces a corresponding sub-optimal LEACH-VF cluster to be used for data transmission.

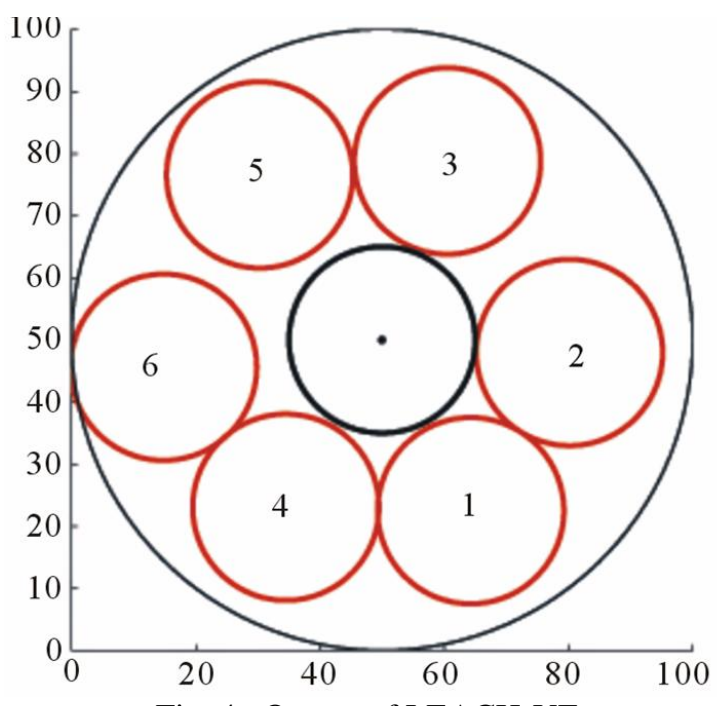

Fig. 4. Output of LEACH-VF

\section{VARIOUS CLUSTERING APPROACHES}

\section{A. LEACH}

The low-energy adaptive clustering hierarchy (LEACH) and another improved centralized LEACH deploy randomized rotation of cluster-heads to evenly distribute the energy load among all sensors in a WSN. This paper proposes an improved LEACH (LEACH-C) algorithm called partition-based LEACH (pLEACH), which firstly partitions the network into optimal number of sectors, and then selects the node with the highest energy as the head for each sector, using the centralized calculations. The simulation results and analysis show that Pleach could achieve much better performance of WSN in terms of the energy dissipation, network lifetime and quality of communication.

\section{B. PEGASIS}

PEGASIS protocol form a chain including all nodes in the network using greedy algorithm so that each nodes transformed to and received from a neighbor. In each round, randomly selected node takes turns to transmit the aggregated information to the base station.. PEGASIS introduces excessive delay for distant nodes on the chain. The single leader can become a bottleneck in PEGASIS. PEGASIS increases network lifetime two-fold compared to the LEACH protocol. Again PEGASIS avoids the formation of clustering overhead of $\mathrm{LEACH}$, but it requires dynamic topology adjustment since sensor energy is not tracked. PEGASIS introduces excessive delay for distant nodes on the chain. [11] The single leader can become a bottleneck in PEGASIS. PEGASIS increases network lifetime two-fold compared to the LEACH protocol.

\section{TEEN and APTEEN}

Nodes in TEEN and APTEEN are designed to respond to sudden changes in the sensed attribute when node exceed a user defined threshold. They assume that position of the base station is fixe and every node in the network directly communicates to base station. TEEN only transmits timecritical data, while APTEEN performs periodic data transmissions. In this respect APTEEN is also better than LEACH because APTEEN transmits data based on a threshold value whereas LEACH transmits data continuously.

LEACH, TEEN, APTEEN and PEGASIS have similar features and their architectures are to some extent similar. [7] They have fixed infrastructure. LEACH, TEEN, APTEEN are cluster based routing protocols, whereas PEGASIS is a chain-based protocol. The performance of APTEEN lies between TEEN and LEACH with respect to energy consumption and longevity of the network [9].

\section{TABLE I PERFORMANCE MEASUREMENTS OF PROTOCOLS}

\begin{tabular}{l|l|l|l|l|l|} 
Protocols & Mobility & \multicolumn{1}{|c|}{$\begin{array}{l}\text { Power } \\
\text { management }\end{array}$} & $\begin{array}{c}\text { Network } \\
\text { lifetime }\end{array}$ & Scalability & $\begin{array}{l}\text { Resource } \\
\text { awareness }\end{array}$ \\
\hline LEACH & Fixed BS & Maximum & Very good & Good & Yes \\
\hline TEEN & Fixed BS & Maximum & Very good & Good & Yes \\
\hline APTEEN & Fixed BS & Maximum & Very good & Good & Yes \\
\hline PEGASIS & Fixed BS & Maximum & Very good & Good & Yes \\
\hline SPIN & Supported & Limited & Good & Limited & Yes \\
\hline DD & Limited & Limited & Good & Limited & Yes \\
\hline
\end{tabular}




\section{IJIREEICE \\ International Journal of Innovative Research in \\ Electrical, Electronics, Instrumentation and Control Engineering \\ ISO 3297:2007 Certified \\ Vol. 5, Issue 3, March 2017}

\section{VI.CONCLUSION}

The routing techniques are classified as proactive, reactive and hybrid, based on their mode of functioning and type of target applications. Further, these are classified as direct communication, flat and clustering protocols, according to the participating style of nodes. Again depending on the network structure, these are categorized as hierarchical, data centric and location based. We have compared various protocols and future work will be focused on saving energy with concept of mobile sinks in various protocols.

\section{REFERENCES}

[1] I.F. Akyildiz, W. Su, Y. Sankara subramaniam, A Survey on Sensor Networks, in IEEE Communications Magazine, 2002, Vol. 40, Issue. 8, pp.102-114.

[2] K. Sohrabi, J. Gao, V. Ailawadhi and G. Pottie, Protocols for Selforganization of a Wireless Sensor Network, in IEEE Personal Communications, Vol. 7, Issue. 5, 2000, pp. 16-27.

[3] Chandrakasan, Amirtharajah, Cho, Goodman, Konduri, Kulik, Rabiner and Wang, Design Considerations for Distributed Microsensor Systems, in IEEE Custom Integrated Circuits Conference (CICC), Vol. 9, Issue. 2, May 1999, pp. 279-286..

[4] Koushik Majumder, Sudhabindu Ray,A Novel Power Efficient Routing Scheme for Wireless Sensor Network, International Journal of Instrumentation, Control \& Automation (IJICA), Vol. 1, Issue. 1, 2011, pp.23-29.

[5] Rakesh Kumar, Fault Tolerance for Chain based Hierarchical Data Gathering Protocol for WSN, International Journal of Engineering Research and Applications (IJERA), Vol. 2, Issue. 4, August 2012, pp.906-911.

[6] Lu Tao1, Zhu Qing-Xin, Zhang Luqiao, an Improvement for LEACH Algorithm in Wireless Sensor Network, IEEE Conference on Consumer Communications and Networking, Vol. 6, Issue. 4, June 2010, pp $593-598$.

[7] Jamal N. Al-Karaki, Routing Techniques in Wireless Sensor Networks: A Survey, IEEE Wireless Communications, Vol. 2, No. 2, December 2004, pp.704-709.

[8] Raju Pal, Ajay K. Sharma, MSEP-E: Enhanced Stable Election Protocol with Multihop Communication, IEEE Conference on AsiaPacific Service Computing Conference, Vol. 2, Issue. 2, February 2011, pp 172- 178.

[9] K.Suganthi, Dr.B.Vinayaga Sundaram, K.S.Vishwa Kumar, J.Syed Ashim, S.Saravana Kumar, Improving Energy Efficiency and Reliability using multiple mobile sinks and hierarchical clustering in Wireless Sensor Networks, IEEE-International Conference on Recent Trends in Information Technology, MIT, Anna University, Chennai, Vol.5, No.4, June 3-5, 2011.

[10] Z. Maria Wang, Stefano Basagni, Emanuel Melachrinoudis and Chiara Petriol, Exploiting Sink Mobility for Maximizing Sensor Networks Lifetime, IEEE Proceedings of the 38th Hawaii International Conference on System Sciences, pp. 1-5, March 2005.

[11] Fahed Awad, Eyad Taqieddin, Asmaa Seyam, Energy-Efficient and Coverage-Aware Clustering in Wireless Sensor Networks, Wireless Engineering and Technology, SciRes, Vol. 3, 2012, pp.142-151. 